\title{
Heat Transfer Performance of Different Fluids During Natural Convection in Enclosures with Varying Aspect Ratios
}

\author{
Rajashekhar Pendyala ${ }^{1,2}$, Suhaib Umer Ilyas ${ }^{2}$, and Yean Sang Wong ${ }^{3}$ \\ ${ }^{1}$ Centre for Process Systems Engineering, Institute of Autonomous Systems, Universiti Teknologi PETRONAS, 32610 Seri Iskandar, \\ Perak Darul Ridzuan, Malaysia \\ ${ }^{2}$ Chemical Engineering Department, Universiti Teknologi PETRONAS, 32610 Seri Iskandar, Perak Darul Ridzuan, Malaysia \\ ${ }^{3}$ Schott Glass (Malaysia) Sdn. Bhd., 2024, Tingkat Perusahaan 6, Zon Perindustrian Bebas 2, 13600 Perai, Penang, Malaysia
}

\begin{abstract}
The heat transfer process takes place in numerous applications through the natural convection of fluids. Investigations of the natural convection heat transfer in enclosures have gained vital importance in the last decade for the improvement in thermal performance and design of the heating/cooling systems. Aspect ratios $(\mathrm{AR}=$ height/length) of the enclosures are one of the crucial factors during the natural convection heat transfer process. The investigated fluids consisting of air, water, engine oil, mercury, and glycerine have numerous engineering applications. Heat transfer and fluid flow characteristics are studied in 3-dimensional rectangular enclosures with varying aspect ratios (0.125 to 150$)$ using computational fluid dynamics (CFD) simulations. Studies are carried out using the five different fluids having Prandtl number range 0.01 to 4500 in rectangular enclosures with the hot and cold surface with varying temperature difference $20 \mathrm{~K}$ to $100 \mathrm{~K}$. The Nusselt number and heat transfer coefficients are estimated at all conditions to understand the dependency of ARs on the heat transfer performance of selected fluids. Temperature and velocity profiles are compared to study the flow pattern of different fluids during natural convection. The Nusselt number correlations are developed in terms of aspect ratio and Rayleigh number to signify the natural convection heat transfer performance.
\end{abstract}

\section{Introduction}

The mechanism of heat transfer persuaded by buoyancy force, which causes movement of fluid due to change in density by temperature gradient, is known as natural convection. This buoyancy-driven heat transfer process in enclosures has a variety of industrial applications in heat transfer processes. These applications involve mainly in thermal management systems for heating or cooling of chemical processes and electronics. Examples of heat transfer process by natural convection includes heat dissipation systems for power transformers, solar energy collectors, heating through electric baseboard heaters, cryogenic storages, double-pane windows, cooling of electronic chips and transistors through heat sink systems, chemical vapor deposition, nuclear power plants and many more. Natural convection heat transfer rates are relatively less than heat transfer through forced convection; however, the natural convection process does not involve any additional energy requirement for pumping, and the heat transfer process is dependent on the geometry and fluid properties.

The mechanism of natural convection heat transfer process has been widely studied over the years due to extensive applicability in the field of energy conservation. Experimental and numerical studies have been carried out in different geometries and scenarios [1-
3]. However, limited studies are available in $3 \mathrm{D}$ vertical enclosures with varying aspect ratios (ARs). Recent literature reports the numerical and experimental investigations for a variety of natural convection scenarios in different geometries such as pin-finned isothermal vertical plate [4], horizontal isothermal cylinders [4], finned enclosures [5], vertical plate microchannel [6], vertical enclosures [7], square enclosures heated from bottom [8] and partially open enclosures [9]. Ganguli et al. [7] investigated the flow patterns during natural convection in air, water, mercury, and silicon oil in vertical slots with the aspect ratio (AR) of 20. Instability mechanism and multicellular patterns were observed in vertical rectangular slots at different Prandtl numbers (Pr). Nishimura et al. [10] studied the natural convection behavior of fluids numerically and experimentally in rectangular enclosures with multiple vertical partitions. The concept was applicable to reduce heat transfer rate up to $70-90 \%$ by introducing $2-5$ partitions between the hot and cold surfaces. In the later studies, Turkoglu and Yücel [11] developed a computerbased SIMPLE algorithm to investigate the dependency of aspect ratios, number of partitions, and Rayleigh number on the heat transfer rate. It was reported that the Nusselt number decreases with increasing partition numbers. It was also observed that the Nusselt number values decrease with increasing aspect ratio.

\footnotetext{
* Corresponding author: rajashekhar_p@utp.edu.my
} 
Long and Zhang [12] experimentally investigated the natural convection behavior of helium at supercritical conditions in a vertical cylinder. It was found that supercritical helium showed better heat transfer performance than conventional gases in vertical, closed cylinders with AR of 27. In another study by Altaç and Kurtul [13], natural convection heat transfer was numerically studied in tilted rectangular enclosures with angle $0 \mathrm{o}-90 \mathrm{o}$. The studies were carried out for Rayleigh number of range 105-107 and aspect ratio of 1 and 2. It was observed that heat transfer rates increase with increasing aspect ratio. Warren et al. [14] described a comprehensive natural convection characteristic in the vertical and horizontal geometries. It was reported that convective heat transfer was improved with decreasing aspect ratios. The cavities with high aspect ratios showed lower Nusselt number values than the squared cavities. Experimental investigations were carried out by Kamotani et al. [15] for enclosures with varying aspect ratios. They found that the Nusselt number is independent of the Prandtl number for $\mathrm{AR}=1$ but highly dependent on the Prandtl number for $\mathrm{AR}<1$. In another numerical study [16], it was observed that the Nusselt number and Rayleigh number are directly proportional to the Prandtl number for laminar conditions in square cavities. Arici et al. [17] investigated heat transfer and fluid flow characteristics in double, triple and quadruple pane windows for a variety of aspect ratios and temperature gradients. In their numerical study, they observed that gap width is an important energy-saving factor, and the effect is more noticeable at lowtemperature gradients. Ganguli et al. [18] investigated the heat transfer behavior of air during natural convection across cold and hot surfaces in enclosed rectangular geometries with a wide range of aspect ratios. The flow pattern, temperature distributions, and velocity profiles were examined using 2-dimensional computational fluid dynamics (CFD) simulations. They found that the flow patterns are highly dependent on the aspect ratios of enclosures.

This research presents the investigation of natural convection behavior of different crucial heat transfer fluids, i.e., air, water, engine oil, mercury, and glycerine. These fluids are encountered in numerous engineering applications. A great number of air-based cooling processes are found in the home, commercial, and industrial appliances. The natural convection by airflow is considered as the most economical heat transfer process. The buoyancy movements in air-cooling systems are much higher than the liquids-based cooling media due to the properties of air. Therefore, accurate simulations of air-cooling processes require proper wall boundary conditions for the velocity components. The typical examples include a hot-air balloon, central heating in buildings, convection in the oven, chimney effect, and ice melting processes. Water-based natural convection heat transfer processes used in various types of heat exchangers, cooling jackets, radiators, and thawing of frozen materials. Due to certain limitations in air-cooling systems, water-based cooling systems have known to have a feasible and economical solution for high heat flux applications due to the high specific heat of the water. The engine oil has less density than water and can operate at higher temperatures $\left(<100^{\circ} \mathrm{C}\right)$ compared to water. The main applications of engine oil include distribution transformer and engine cooling management. The two other fluids chosen in this research are glycerine and mercury, which have a critical significance in the chemical and nuclear industries. The knowledge of natural convection behavior is a crucial aspect for the storage, transportation, and processing of these fluids.

Limited investigations are carried out on the fluid flow and heat transfer characteristics using 3dimensional CFD simulations. The natural convection heat transfer in enclosures of the fluids have significance towards numerous engineering application, and a wide research gap is found in the literature. 3-Dimensional models of rectangular vertical enclosures can predict natural convection behavior with more accuracy and reliability than 2-Dimensional models due to edge effects of the enclosure. CFD analyses are performed to investigate fluid flow and heat transfer characteristics for a wide range of aspect ratios. The Nusselt number and heat transfer coefficients for each fluid at varying conditions are estimated and compared. A generic Nusselt number correlation is proposed for different fluids, which is based on the simulated results.

\section{Methodology}

Heat transfer and fluid flow characteristics are numerically studied during natural convection heat transfer for five different fluids in the rectangular enclosures. The geometry consists of a rectangular enclosure with one hot and one cold surface, shown in Fig. 1. Insulated properties are implied to the other four surfaces of the enclosure. Heat transfer performance for five different fluids is investigated, which are having comparable properties with air, water, engine oil, mercury, and glycerine, respectively. All fluids are present in the ANSYS database, and the built-in thermophysical properties of the fluids are used in the simulation. The studied fluids are having Prandtl number range of $0.5 \leq \operatorname{Pr} \leq 4500$. Simulations are performed at varying aspect ratios $(0.125 \leq \mathrm{AR} \leq 150)$ and temperature difference $(20 \mathrm{~K} \leq \Delta \mathrm{T} \leq 100 \mathrm{~K})$ across the hot and cold surface. The lengths $(\mathrm{L})$ and the heights $(\mathrm{H})$ of the enclosures are varied to get 18 geometries with different aspect ratios in the range of 0.125 to 150 . The width (W) of the enclosure is kept constant for studied geometries. The CFD simulations are performed in ANSYS (Fluent). Fundamental equations and literature are used to obtain thermophysical data for all fluids [19, 20]. During numerical calculations, all fluids are assumed as a single phase. For better accuracy, fine meshing is used near the edges of the rectangular geometry with bias meshing technique. The Boussinesq approximation is used, which implies that the fluid movement is mainly due to buoyancy force. The steadystate simulations are performed with the acceleration of gravity in the y-direction (negative). Pressure based solver is applied along with the absolute velocity 
formulation. Energy equation, laminar model equations, and surface-to-surface equations are considered. For buoyancy calculations, the Body force weighted method is implied for pressure-based solutions. Convergence criteria is set for scaled residuals of energy, velocity, and continuity models. For discretization of momentum and energy, First-order upwind and Third-order Monotone Upstream centered Schemes for Conservation Laws (MUSCL) are applied.

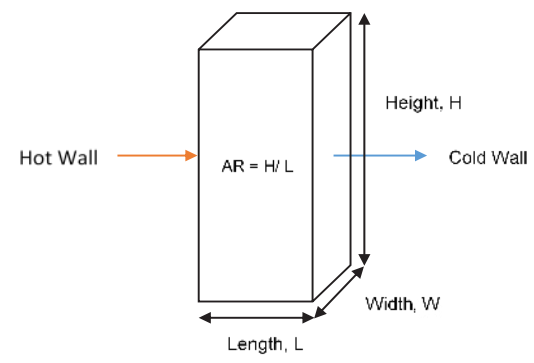

Fig. 1. The rectangular enclosure under consideration with the hot and cold surface.

\section{Results and discussion}

\subsection{Flow patterns at varying ARs}

Numerical studies are carried out for the investigation of the fluid flow behavior of different fluids at varying temperature gradients. The simulations are performed for all aspect ratios of the rectangular enclosures. It is observed that the aspect ratio has a significant impact on the average velocity of fluid during natural convection. An increase in aspect ratio leads to intensifying the average velocity of the fluid. Velocity contours at isosurface of five fluids are presented in Fig. 2 at a low aspect ratio (0.125), and a temperature difference of 80 $\mathrm{K}$. Contours with different scales are compared for the complete understanding of the flow patterns and velocity distribution generated in various fluids. Comparative velocity contours at iso-surface of fluids during natural convection in enclosures at higher aspect ratios are presented in Fig. 3.

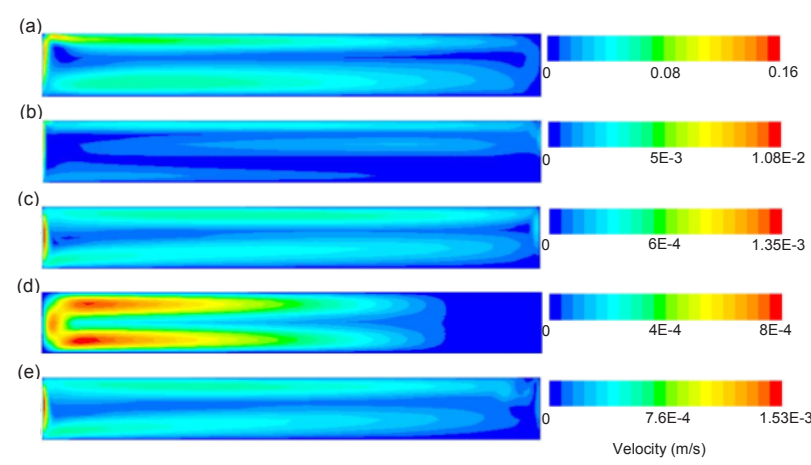

Fig. 2. Velocity distribution in rectangular enclosures at $A R=$ 0.125 and $\Delta \mathrm{T}=80 \mathrm{~K}$ for (a) Air (b) Water (c) Engine oil (d) Mercury and (e) Glycerine.

It is observed that, in all cases, air possesses a higher average velocity during natural convection as compared to other fluids. Water, engine oil, mercury, and glycerine exhibit lower values of velocities due to high viscosity than air. It is attributed that the fluid movement is highly dependent on the viscosity of fluids. The flow regimes in rectangular enclosures indicate the flow behavior of fluids. Heat transfer characteristics are mainly governed by the flow regimes which are generated by the fluid movement during natural convection in enclosures. The formation of multicellular flow is observed in most of the conditions in rectangular enclosures with low aspect ratios. Multiple cells with unequal distribution illustrate that the heat transfer process in rectangular enclosures is dominant by convective heat transfer. The flow regimes with multicellular flow patterns are not observed in aspect ratios with higher values. In some cases, increasing the aspect ratio of the enclosure leads to diminishing multicellular flow regimes, and formed unicellular flow. Flow regimes with multicellular patterns exhibit better convective heat transfer characteristics compared to unicellular pattern [18].

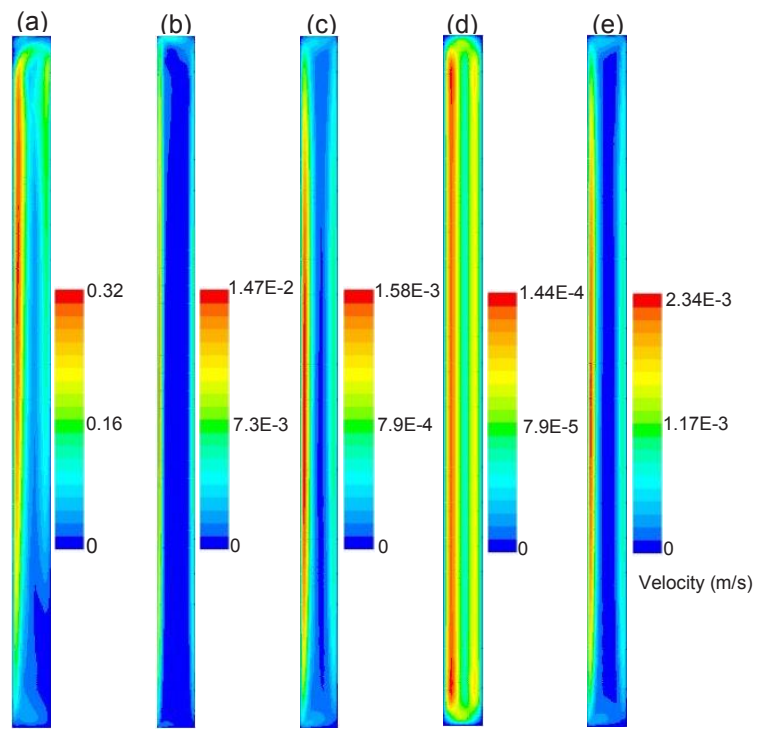

Fig. 3. Velocity distribution in rectangular enclosures at $A R=$ 18 and $\Delta \mathrm{T}=80 \mathrm{~K}$ for (a) Air (b) Water (c) Engine oil (d) Mercury and (e) Glycerine.

\subsection{Heat transfer characteristics at varying ARs}

The Nusselt numbers and heat transfer coefficients are estimated for different fluids at varying aspect ratios and temperature differences. It is found that the heat transfer characteristics are significantly influenced by the height and length of the rectangular enclosures. Heat transfer coefficients are observed to be higher in the enclosures with high aspect ratios for all fluids, shown in Fig. 4. However, the Nusselt number values are found to be decreasing as the aspect ratio of the enclosure is increased, shown in Fig. 5. This behavior is attributed to the conductive dominant heat transfer characteristics of the fluid at high aspect ratios. This phenomenon was described extensively by Ganguli et al. [18]. They concluded that better heat transfer performance is obtained in enclosures with low aspect ratios due to multicellular flow pattern formation. 

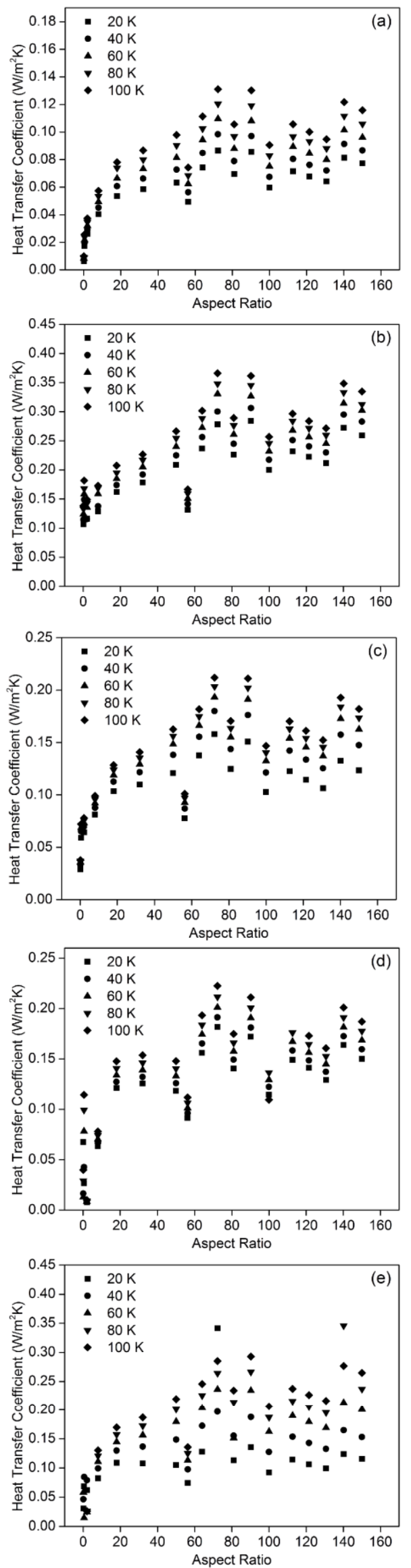

Fig. 4. Heat transfer coefficient of fluids with varying aspect ratios at different temperature gradients for (a) Air (b) Water (c) Engine oil (d) Mercury and (e) Glycerine.
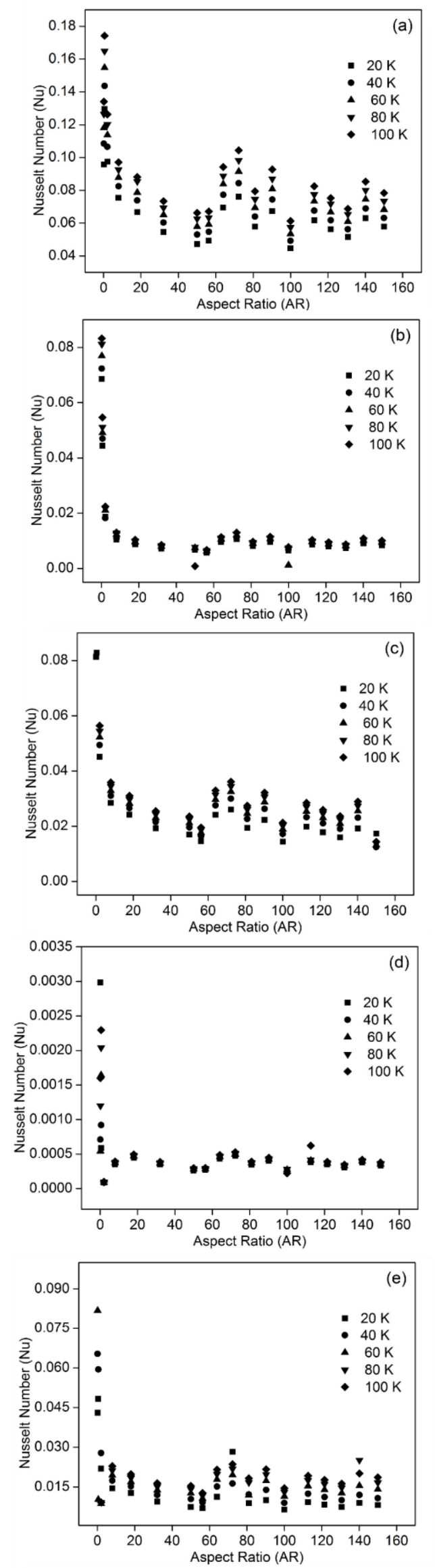

Fig. 5. Nusselt number of fluids with varying aspect ratios at different temperature gradients for (a) Air (b) Water (c) Engine oil (d) Mercury and (e) Glycerine. 
Heat transfer properties of different fluids are estimated and analyzed in the middle of the rectangular enclosure $(0.025 \mathrm{~m})$ by creating an iso-surface on $\mathrm{z}$-coordinate. The impact of the enclosure's size on the Nusselt number is extensively analyzed for different fluids. It is observed that, besides aspect ratio, the Nusselt number values are highly dependent on the temperature between the hot and cold walls and thermophysical properties of the fluid. The effect of aspect ratio on Nusselt number is shown in Fig. 5. The Nusselt number values are observed to be increasing with an increase in temperature gradient at a constant aspect ratio. Mercury possesses the lowest Nusselt number values as compared to other fluids. Water shows the highest heat transfer coefficient values at all aspect ratios. Heat transfer coefficients of different fluids are mainly governed by flow regimes that are generated in rectangular enclosures during natural convection. There are three categories of flow regimes of fluids: conduction flow regime, transition flow regime, and boundary layer flow regime [18]. The low aspect ratio enclosures (0.125 and 0.5) exhibit high Nusselt number due to conductive dominant heat transfer, shown in Fig. 5. Temperature distribution contours for different fluids during natural convection are presented in Fig. 6 and Fig. 7 for low and high aspect ratios, respectively. It is observed that heat transfer in rectangular enclosures with the low aspect ratio (0.5) falls in boundary layer flow regimes, shown in Fig. 6. In this type of flow regime, most of the fluid exhibits conductive heat transfer and only thin boundary layers near the wall surface show conductive heat transfer. Similar behavior is observed for five fluids in low aspect ratios during natural convection. Temperature distribution in the enclosure with an aspect ratio of 18 for different fluids is shown in Fig. 7.

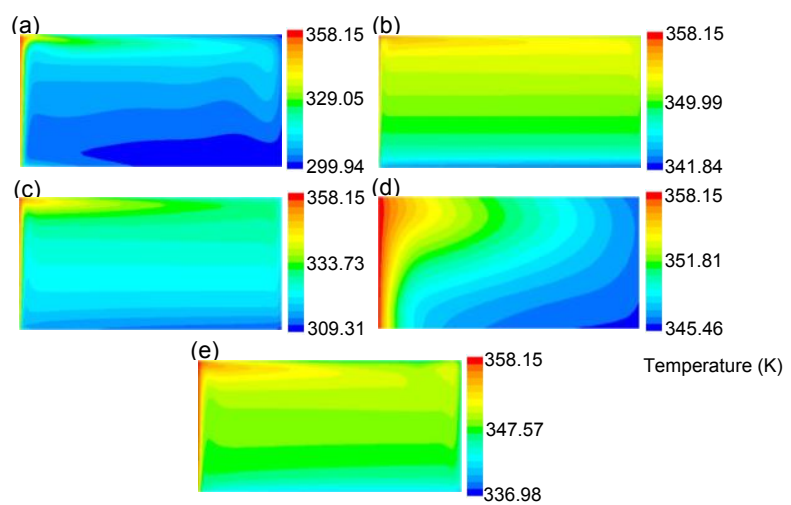

Fig. 6. Temperature distribution of different fluids in rectangular enclosures with $\mathrm{AR}=0.5$ and $\Delta \mathrm{T}=60 \mathrm{~K}$ for (a) Air (b) Water (c) Engine oil (d) Mercury and (e) Glycerine.

It is observed that heat transfer behavior, in most cases, in this enclosure follows the transition flow regime. It is attributed to the increase in conductive heat transfer near the boundaries of the wall and hence decreasing the convective heat transfer and Nusselt number values. Similar behaviors were observed in the studies by Ganguli et al. [18]. The heat transfer process in enclosures with high aspect ratios is governed mainly by conduction. Linear temperature distribution can be observed from temperature contours, which cause a reduction in the Nusselt number. At very high aspect ratios, the distance between the hot and cold surface is very low, which causes complete domination of conductive heat transfer. Similar heat transfer behavior is observed for all the fluid under consideration. Improvement in convective heat transfer is observed at the condition with higher Rayleigh numbers. Similar trends of conductive and convective heat transfer during natural convection in enclosures with low and high aspect ratios were obtained in previous studies $[14,18]$.

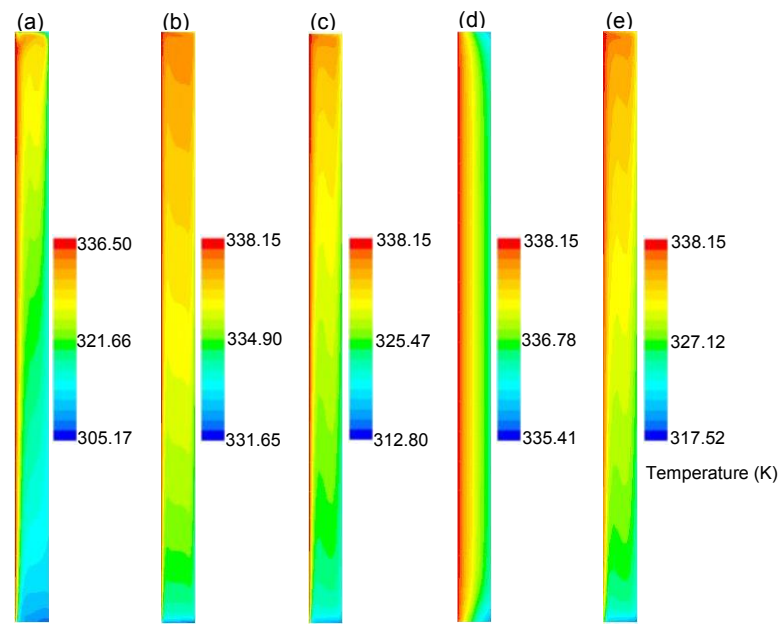

Fig. 7. Temperature distribution of different fluids in rectangular enclosures with $\mathrm{AR}=18$ and $\Delta \mathrm{T}=40 \mathrm{~K}$ for (a) Air (b) Water (c) Engine oil (d) Mercury and (e) Glycerine.

\subsection{Correlation for Nusselt number}

Extended simulations are performed for different aspect ratios than previous work [21] to obtain complete data sets for the Nusselt number and the Rayleigh number for five different fluids. Correlations are developed for the Nusselt number as a function of Rayleigh number and aspect ratio. The proposed correlation for different fluids is given in eq. 1 .

$$
\mathrm{Nu}=\mathrm{a}_{1}(\mathrm{AR})^{\mathrm{a} 2}(\ln \mathrm{Ra})^{\mathrm{a} 3}
$$

The correlation is proposed for five different fluids with varying temperature differences between the hot and cold surface $(20 \mathrm{~K} \leq \mathrm{T} \leq 100 \mathrm{~K})$, Prandtl number $(0.01 \leq \mathrm{Pr}$ $\leq 4500)$ and aspect ratios $(8 \leq \mathrm{AR} \leq 150)$. Correlation coefficients, Average Absolute Deviation (AAD) and the Sum of Squared Errors (SSE) for different fluids are tabulated in Table 1. Parity plots are presented in Fig. 8 to examine the performance of correlation.

Table 1. Correlation coefficients, Average Absolute Deviation (AAD) and Sum of Squared Errors (SSE) and aspect ratio ranges for different fluids.

\begin{tabular}{cccccc}
\hline \multirow{2}{*}{ Fluids } & \multicolumn{6}{c}{ Correlation coefficients } & AAD (\%) & SSE \\
\cline { 2 - 6 } & $\mathrm{a}_{1}$ & $\mathrm{a}_{2}$ & $\mathrm{a}_{3}$ & & \\
\hline Air & $1.46 \mathrm{E}-5$ & 0.19 & 3.23 & 11.33 & $1.5 \mathrm{E}-2$ \\
Water & $7.52 \mathrm{E}-7$ & -0.014 & 3.31 & 18.28 & $4 \mathrm{E}-4$ \\
oil & $7.57 \mathrm{E}-6$ & 0.0256 & 3.114 & 14.81 & $4.3 \mathrm{E}-3$ \\
Mercury & $2.71 \mathrm{E}-7$ & 0.48 & 2.65 & 19.58 & $6.2 \mathrm{E}-7$ \\
Glycerine & $1.53 \mathrm{E}-6$ & 0.15 & 3.02 & 15.28 & $9.7 \mathrm{E}-4$ \\
\hline
\end{tabular}




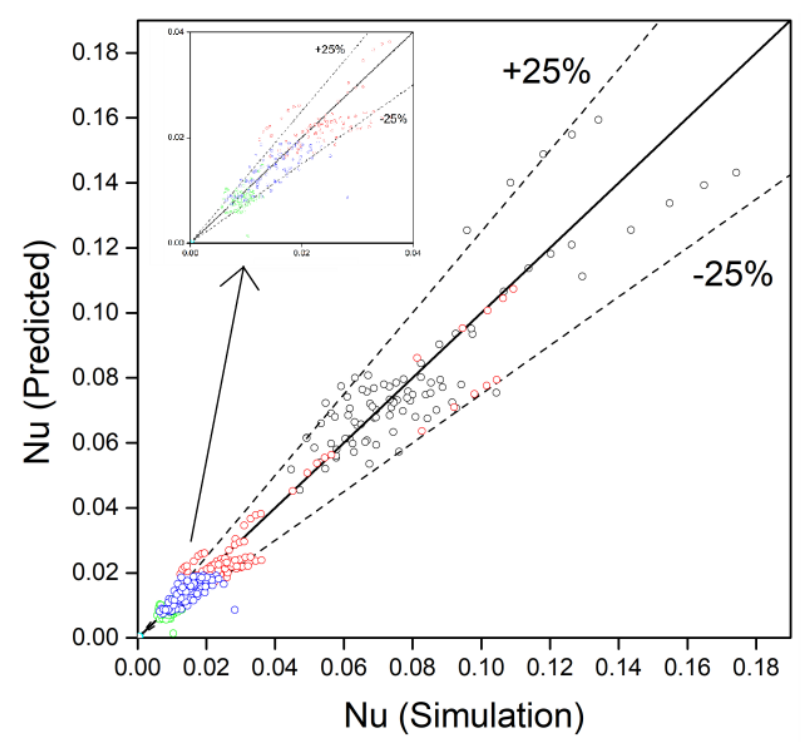

Fig. 8. Parity plot of Nusselt number correlation (Eq. 1) for all fluids, i.e., air (black), water (green), engine oil (red), mercury (cyan) and glycerine (blue).

\section{Conclusions}

Heat transfer performance of five different fluids is studied during natural convection at varying aspect ratios of rectangular enclosures. Numerical studies are performed at varying temperature gradients between the hot and cold walls. It is found that aspect ratio has a significant impact on the natural convection heat transfer characteristics in the enclosures. Rectangular enclosures with lower aspect ratios are observed to have better heat transfer characteristics due to the formation of multicellular flow regimes. Enclosures with high aspect ratios are found to be conductive dominant fluid regimes and exhibit lower values of the Nusselt number. The Nusselt number is estimated for all cases, and correlations are developed. Improved convective heat transfer performance is observed at high Rayleigh number conditions.

\section{Acknowledgment}

This work is supported by the Chemical Engineering Department, Universiti Teknologi PETRONAS. The financial assistance is provided by YUTP (Grant No. 015LC0-271).

\section{References}

1. M. Arshad, M.H. Inayat, I.R. Chughtai, App. Therm. Eng. 31, 20-27 (2011)

2. A. Gowrishankar, S. Vignesh, P.B. Shyam Sunder, R. Abhinav, M. Vivek, V. Ratna Kishore, Heat Trans. Asian Res., 44, 620-640 (2015)

3. A.K.A. Shati, S.G. Blakey, S.B.M. Beck, App. Therm. Eng., 51, 364-370 (2013)

4. L.K. Sahoo, M.K. Roul, R.K. Swain, Heat Trans. Asian Res., 46, 840-862(2017)
5. N. Kasayapanand, App. Therm. Eng., 29, 131-141 (2009)

6. M. Narahari, R. Pendyala, App. Mech. Mater., 705, 182-187 (2015)

7. A. Ganguli, A. Pandit, J. Joshi, Chem. Eng. Sci., 62, 4479-4495 (2007)

8. B. Calcagni, F. Marsili, M. Paroncini, App. Therm. Eng., 25, 2522-2531 (2005)

9. B. Gera, R. Singh, Heat Trans. Asian Res., 44, 154171 (2015)

10. T. Nishimura, M. Shiraishi, F., Nagasawa, Y. Kawamura, Int. J. Heat Mass Trans., 31, 1679-1686 (1988)

11. H. Turkoglu, N. Yücel, Heat Mass Trans., 32, 1-8 (1996)

12. Z.Q. Long, P. Zhang, Cryogenics, 61, 120-126 (2013)

13. Z. Altaç, Ö. Kurtul, App. Therm. Eng., 27, 18321840 (2007)

14. M.R. Warren, P.H. James, I.C. Young, Handbook of Heat Transfer, McGraw-Hill Education, Access Engineering, (1998)

15. Y. Kamotani, S. Ostrach, L. Wang, AIAA J., 21, 290-294 (1983)

16. T. Pesso, S. Piva, Int. J. Heat Mass Trans., 52, 10361043 (2009)

17. M. Arıc1, H. Karabay, M. Kan, Energ. Buildings, 86, 394-402 (2015)

18. A. Ganguli, A. Pandit, J. Joshi, Chem. Eng. Res. Des., 87, 711-727 (2009)

19. A. Bejan, Convection Heat Transfer (3rd Ed.), John Wiley \& Sons, New York, (2004)

20. F.P. Incropera, D.P. Dewitt, Fundamentals of Heat and Mass Transfer (5th Ed.). New York: Wiley \& Sons, (2002)

21. R. Pendyala, Y.S. Wong, S.U. Ilyas, Chem. Eng. Trans., 45, 793-798 (2015) 